\title{
CAPACIDADE FUNCIONAL E LINGUAGEM DE IDOSOS NÃO-PARTICIPANTES E PARTICIPANTES DE GRUPOS DE INTERVENÇÃO MULTIDISCIPLINAR NA ATENÇÃO PRIMÁRIA À SAÚDE
}

\section{Functional ability and language of participant and non-participant elderly in groups of multidisciplinary intervention in primary health care}

\author{
Aluísia Guerra Albuquerque ("), Giselle Simas Montarroyos de Oliveira ${ }^{(2)}$, \\ Vanessa de Lima Silva ${ }^{(3)}$, Cynthia Barboza do Nascimento ${ }^{(4)}$
}

\section{RESUMO}

Objetivo: verificar e comparar a capacidade funcional e a linguagem de idosos participantes e não-participantes de grupos com intervenção multidisciplinar na atenção primária à saúde. Método: participaram do estudo 60 indivíduos maiores de 60 anos ou mais de ambos os sexos, residentes na área de cobertura de duas Unidades de Saúde da Família do Município de Camaragibe/PE. O estudo foi descritivo, observacional, transversal e foi realizado no período de maio a setembro de 2010. Para a coleta dos dados foi utilizado um questionário socioeconômico e demográfico pré-estruturado, além dos protocolos de avaliação das Atividades Instrumentais de Vida Diária e o Mini-Exame de Estado Mental, para avaliação da linguagem. Resultados: a maioria dos idosos avaliados encontra-se na faixa etária de 60-69 anos, é do sexo feminino, da raça/cor branca, casado(a), analfabeto, com renda de um a dois salários mínimos. Na avaliação da capacidade funcional de idosos participantes e não-participantes de grupos revelou que $90 \%$ e $80 \%$ são independentes em relação às atividades instrumentais de vida diária, respectivamente. Em relação à linguagem, 10\% dos idosos participantes de grupo apresentaram déficit contra apenas $6,7 \%$ dos idosos não-participantes. Conclusão: há necessidade de estudos com amostras populacionais maiores na tentativa de comprovar os efeitos positivos das atividades de grupo, como diz a literatura, e ainda realizar novos estudos sobre a influência da linguagem na capacidade funcional dos idosos, a fim de encontrar relevância estatística dos dados e assim poder planejar e melhorar o atendimento às necessidades e os problemas decorrentes do envelhecimento.

DESCRITORES: Idoso; Linguagem; Atenção Primária à Saúde

(') Fonoaudióloga; Funcionária Pública da Prefeitura Municipal de Vertentes-PE; Especialista em Fonoaudiologia na Saúde Pública-FOP/UPE; Mestranda em Saúde Humana e Meio Ambiente-CAV/UFPE.

(2) Fonoaudióloga; Docente do Serviço Nacional de Aprendizagem Comercial-SENAC/PE, Coordenadora da Especialização em Fonoaudiologia na Saúde Pública;

Especialista em Linguagem-UNICAP/PE e em Fonoaudiologia na Saúde Pública-FOP/UPE.

(3) Fonoaudióloga; Professora do curso de Especialização em Fonoaudiologia na Saúde Pública da Universidade de Pernambuco; Mestre em Saúde Coletiva pela Universidade Federal de Pernambuco - UFPE, Doutoranda em Saúde Pública pelo Centro de Pesquisas Aggeu Magalhães CpqAM-FIOCRUZ.

\section{INTRODUÇÃO}

Diante da realidade inquestionável das transformações demográficas iniciadas no último século e que nos fazem observar uma população cada vez mais envelhecida, evidencia-se a importância de garantir aos idosos não só uma sobrevida maior, mas também uma boa qualidade de vida ${ }^{1}$.

(4) Fonoaudióloga do Núcleo de Apoio à Saúde da Família do município de Camaragibe-PE; Mestre e Doutoranda em Saúde Pública pelo Centro de Pesquisas Aggeu MagaIhães - CpqAM-FIOCRUZ.

Conflito de interesses: inexistente 
A expectativa de vida no Brasil vem aumentando consideravelmente, estima-se que em 2050 o número de idosos seja maior do que o de crianças abaixo de 15 anos $^{2}$.

Em virtude do aumento do número de idosos, surge a necessidade de políticas e ações voltadas para esta faixa etária, pois os mesmos chegam a tal idade com limitações, doenças e agravos crônicos não transmissíveis, que requerem acompanhamento constante. Estas manifestações crônicas tendem a surgir de forma mais acentuada na idade avançada e frequentemente estão mais associadas à comorbidades, gerando um processo que vem a prejudicar a capacidade do idoso em se comunicar, e limitando suas atividades diárias. Ainda que não sejam fatais, essas limitações tendem a comprometer a qualidade de vida desses idosos.

O conceito de qualidade de vida está relacionado à autoestima e ao bem-estar pessoal; abrange uma série de aspectos como a capacidade funcional, o nível socioeconômico, o estado emocional, a interação social, a atividade intelectual, o autocuidado, o suporte familiar, o próprio estado de saúde, os valores culturais, éticos e a religiosidade, o estilo de vida, a satisfação com o emprego e/ou com atividades diárias e o ambiente em que se vive ${ }^{1}$.

A Política Nacional de Saúde do Idoso tem como propósito "a promoção do envelhecimento saudável, a manutenção e a melhoria, ao máximo, da capacidade funcional dos idosos, a prevenção de doenças, a recuperação da saúde dos que adoecem e a reabilitação daqueles que venham a ter a sua capacidade funcional restringida" ${ }^{\text {. }}$

Com o crescimento do contingente de idosos e o prolongamento da expectativa de vida, a preocupação em relação à capacidade funcional vem aumentando em diversos campos do conhecimento. $\mathrm{Na}$ área da saúde, a avaliação da capacidade funcional é essencial para a escolha do melhor tipo de intervenção e monitorização do estado clínico-funcional dos idosos ${ }^{4}$.

A diminuição das capacidades sensório-perceptivas, que ocorre no processo de envelhecimento, pode afetar a comunicação das pessoas idosas. A deficiência auditiva, por exemplo, gera um dos mais incapacitantes distúrbios de comunicação, impedindo-o de desempenhar plenamente seu papel na sociedade, tais alterações poderão levar o idoso ao isolamento familiar e social ${ }^{2}$.

A complexidade do perfil de morbimortalidade do idoso requer atenção especial dos serviços de saúde. Dentro deste contexto o trabalho das equipes de saúde da família, bem como de todos os profissionais atuantes na atenção básica devem ser voltados também para essa população que vem crescendo cada vez mais.
Em 1994, o Ministério da Saúde adotou a Saúde da Família como uma estratégia prioritária para a organização da Atenção Básica e estruturação do sistema de saúde. A Saúde da Família trabalha com práticas interdisciplinares desenvolvidas por equipes que se responsabilizam pela saúde da população a ela adscrita e na perspectiva de uma atenção integral humanizada, considerando a realidade local e valorizando as diferentes necessidades dos grupos populacionais 5 .

$A$ atenção à saúde do idoso na Atenção Básica e Saúde da Família, deve consistir em um processo diagnóstico multidimensional; esse diagnóstico deve ser compreendido em todos os aspectos biológicos, psíquicos, funcionais e sociais, as relações do idoso com a família e com os profissionais de saúde. Deve-se oferecer ao idoso uma atenção humanizada com orientação, acompanhamento e apoio domiciliar, sendo importantes estas ações, pois influenciarão no envelhecimento ativo².

A política pública para a saúde dos idosos preconiza a garantia de atenção integral à saúde da população idosa, enfatizando o envelhecimento saudável e ativo, para isso existe a necessidade de profissionais com formação mais direcionada ao idoso que necessita de cuidados diferentes dos dispostos a outros grupos da população ${ }^{6}$.

Em 2002, o Ministério da Saúde propôs a implantação de Redes Estaduais de Assistência à Saúde do Idoso (portaria MS/GM 702/02) ${ }^{7}$ e Centros de Referência em Atenção à Saúde do idoso (portaria MS/SAS 249/02) ${ }^{8}$, localizados em hospitais, ambulatórios especializados, hospital-dia e assistência domiciliar, que tem como objetivo a manutenção da autonomia e capacidade funcional, a assistência as necessidades de saúde dos idosos e a reabilitação.

No contexto da Atenção Primária à Saúde, uma importante estratégia para a otimização das ações de atenção à saúde do idoso foi a criação dos Núcleos de Apoio à Saúde da Família - NASF, por meio da portaria GM no 154, de 24 de Janeiro de 2008. Os NASF visam apoiar a Estratégia Saúde da Família na rede de serviços e ampliar a abrangência e o escopo das ações da Atenção Primária, bem como sua resolubilidade e devem ser constituídos por equipes compostas por profissionais de diferentes áreas de conhecimento, para atuarem em parceria com os profissionais das equipes das Unidades de Estratégia Saúde da Família ${ }^{9}$.

A atuação da Fonoaudiologia no sistema de saúde é extensa, perpassando diferentes níveis de assistência. A atuação do fonoaudiólogo no NASF junto à Estratégia Saúde da Família (ESF) é ainda um desafio; porém já se sabe que muitos dos trabaIhos realizados no território com as equipes das unidades devem ser realizados junto às escolas e 
a população, promovendo a cultura da promoção e da prevenção da saúde. A Fonoaudiologia realiza palestras, oficinas, grupos de orientação e encaminha casos nos quais é identificada a necessidade de reabilitação para clínicas credenciadas do município ${ }^{10}$.

A Atenção Primária busca atingir seus objetivos trabalhando com grupos, na expectativa de promover o aumento da consciência crítica por intermédio da troca de idéias entre os sujeitos ${ }^{11}$. A estratégia de grupos pode contribuir na promoção, proteção e controle de doenças. Constata-se que, em consultas individuais, o oferecimento de orientações e o desenvolvimento do processo de educação em saúde são insatisfatórios, sendo as reuniões um espaço complementar de troca de informações e estímulo social ${ }^{12}$.

Na saúde coletiva, o grupo é uma estratégia de trabalho e ao mesmo tempo de investigação das condições de populações de usuários do Sistema Único de Saúde. Vários grupos de promoção com crianças, gestantes, idosos são formados para oferecer educação em saúde, com o objetivo de criar ambientes favoráveis ao desenvolvimento humano pela adoção de hábitos e estilos de vida saudáveis que tragam mais qualidade de vida à população ${ }^{13}$.

Reuniões de equipe, oficinas, programas, grupos de orientação, grupos de caminhadas, atendimentos domiciliares ou mesmo intervenções na rua, praças e parques tão comuns à rotina dos serviços de saúde, são práticas grupais e fazem parte de um conjunto de estratégias e tecnologias de cuidado à saúde e, portanto, são consideradas práticas clínico-terapêuticas ${ }^{14}$.

As atividades de grupos facilitam o exercício da autodeterminação e da independência, pois podem funcionar como rede de apoio que mobiliza as pessoas na busca de autonomia e sentido para a vida e na melhora da autoestima ${ }^{15}$. Diante destes benefícios, a formação de grupos de convivência de idosos vem sendo utilizada para proporcionar- Ihes a preservação da capacidade funcional que mantém a autonomia e a independência, mesmo que com limitações.

É nesse contexto que a avaliação da capacidade funcional torna-se essencial para determinar a efetividade e a eficiência das intervenções propostas nos grupos de convivência de idosos, assim como a avaliação da linguagem, aspectos estes fundamentais para uma boa qualidade de vida dos idosos.

O trabalho em grupo é uma realidade no cotidiano das equipes de Atenção Primária/Estratégia Saúde da Família, assim o objetivo deste estudo foi verificar e comparar a capacidade funcional e a linguagem de idosos não-participantes e participantes de grupos com intervenção multidisciplinar na Atenção Primária à Saúde.

\section{MÉTODO}

Trata-se de um estudo descritivo, observacional, transversal, realizado no município de Camaragibe (PE), no período de maio a setembro de 2010. A escolha da área de estudo pautou-se na existência de grupos de convivência de idosos há pelo menos dois anos em funcionamento, formados pelo Núcleo de Apoio à Saúde da Família (NASF). Convém esclarecer que os trabalhos no grupo são realizados de forma integrada entre profissionais do NASF (Fonoaudióloga, Fisioterapeuta, Nutricionista, Psicólogo e Psiquiatra) e profissionais da equipe de Saúde da Família (ESF), sendo planejado e desenvolvido de acordo com a realidade do território. Os encontros dos grupos são mensais, as atividades coletivas, realizadas junto aos idosos, abordam conteúdos que visam fortalecer o auto-cuidado, a promoção à saúde e a prevenção de doenças. Essas ações são realizadas junto a ESF, com foco na intersetorialidade e no fortalecimento da participação da comunidade. Nesse espaço são realizados trabalhos voltados para a Saúde e Cidadania, acontecem oficinas, discussões dos processos saúde-doença, atividades de estímulo das habilidades motoras e cognitivas, buscando sempre a interação e reinserção social dos idosos.

A população do estudo foi constituída de 60 indivíduos com idade de 60 anos e mais, de ambos os sexos, residentes na área de cobertura da Unidade de Saúde da Família de São João São Paulo e da Unidade de Saúde da Família Paulo Afonso. Fizeram parte da coleta, idosos participantes de grupos de intervenção multidisciplinar (G1) e idosos não-participantes (G2). Foi incluído no G1 todos os idosos que participavam do grupo há, pelo menos, dois anos, totalizando 30 idosos. A seleção da amostra do G2 foi aleatória: os idosos não-participantes foram sorteados nas duas unidades de saúde, onde foi determinado a quantia de 30 idosos não- participantes, de forma proposital para efeitos de comparação entre os mesmos; foram excluídos da pesquisa os idosos acamados, demênciados ou portadores de morbidades que afetam suas capacidades físicas e cognitivas.

Para a coleta dos dados foi elaborado um questionário socioeconômico e demográfico pré-estruturado, contendo como não-variável dependente nem independente para o estudo: o sexo, a idade, a escolaridade, a renda, a quantidade de residentes no domicílio e o estado civil, além dos protocolos de avaliação das Atividades Instrumentais de Vida Diária (AIVD) ${ }^{16}$ e o Mini-Exame de Estado Mental (MEEM) ${ }^{16,17 .}$

As Atividades Instrumentais de Vida Diária foram utilizadas como parâmetro para a avaliação 
da capacidade funcional e estão relacionadas com atividades de cuidado com a casa, familiares dependentes e administração do ambiente (limpar a casa, cuidar da roupa, da comida, usar equipamentos domésticos, fazer compras, usar transporte pessoal ou público, controlar a própria medicação e finanças). Por meio do protocolo utilizado na avaliação das Atividades Instrumentais de Vida Diária foi possível determinar se a pessoa idosa é ou não capaz de manter uma vida independente, mantendo assim sua capacidade funcional. Convém dizer que na classificação das Atividades Instrumentais de Vida Diária foi utilizada a escala de Lawton e Brody, adaptada ao contexto brasileiro por Santos e Virtuoso ${ }^{4}$, que apresentam três classificações: dependência total $<5$, dependência parcial $>5<21$ e independência $>21$.

Foi utilizado o MEEM para verificar a linguagem; sabe-se que esse teste busca avaliar a orientação temporal e espacial, memória imediata, atenção e cálculo, evocação, linguagem e praxia. A parte referente à linguagem compreende nomeação, repetição, ordens e cópia. A escala utilizada para análise da linguagem foi proporcional à escala de todo o MEEM, sendo verificada sua validade com significância estatística $p<0,05$.

Este estudo foi aprovado pelo Comitê de Ética em Pesquisa envolvendo seres humanos do CISAM-UPE, de acordo com a resolução $\mathrm{N}$. 으 196/96 do Conselho Nacional de Saúde com o parecer № 015/010. Ressalta-se que os sujeitos da pesquisa assinaram um Termo de Consentimento Livre e Esclarecido, em que se explicavam os objetivos, benefícios e riscos, além da garantia de sigilo de identidade e autonomia para participação. Os idosos que não sabiam ler nem escrever tiveram seu termo lido pelo entrevistador e realizaram a assinatura digital.

Os dados foram analisados pelo solfware Epilnfo 2000, por meio de testes de estatística descritiva (frequência absoluta e relativa) e inferencial (intervalos de confiança). A análise comparativa entre os grupos foi realizada utilizando-se testes qui-quadrado e exato de Fisher, considerando o nível de significância estatística de 5\%.

\section{RESULTADOS}

Segundo as variáveis demográficas foi observada uma prevalência de idosos na faixa etária de $60-69$ anos (60\% entre os que participam de grupo e 53,3\% entre os que não participam).

Comparando os grupos, a única variável que apresentou diferença estatisticamente significante foi a variável sexo. Em ambos os grupos a maioria dos idosos é do sexo feminino, no entanto houve uma diferença em relação ao sexo masculino $-40 \%$ dos idosos participantes de grupo são homens contra $16,7 \%$ dos idosos não participantes (Tabela 1).

Tabela 1 - Distribuição percentual de idosos segundo variáveis demográficas, Camaragibe, 2010

\begin{tabular}{|c|c|c|c|c|c|c|c|}
\hline \multirow[t]{2}{*}{ VARIÁVEL } & \multicolumn{3}{|c|}{ PARTICIPANTE DE GRUPO } & \multicolumn{3}{|c|}{$\begin{array}{l}\text { NÃO PARTICIPANTE DE } \\
\text { GRUPO }\end{array}$} & \multirow{2}{*}{$\begin{array}{l}\text { VALOR } \\
\text { DE P }\end{array}$} \\
\hline & $\mathbf{n}$ & $\%$ & I.C. $(95 \%)$ & $\mathbf{n}$ & $\%$ & I.C. $(95 \%)$ & \\
\hline \multicolumn{8}{|l|}{ SUBRUPO ETÁRIO } \\
\hline 60-69 anos & 18 & 60,0 & $40,6-77,3$ & 16 & 53,3 & $34,3-71,7$ & $0,20^{*}$ \\
\hline 70-79 anos & 12 & 40,0 & $22,7-59,4$ & 11 & 36,7 & $19,9-56,1$ & \\
\hline $80-89$ anos e mais & 0 & 00,0 & - & 3 & 10,0 & $2,1-26,5$ & \\
\hline \multicolumn{8}{|l|}{ SEXO } \\
\hline Masculino & 12 & 40,0 & $22,7-59,4$ & 5 & 16,7 & $5,6-34,7$ & $0,04^{\star *}$ \\
\hline Feminino & 18 & 60,0 & $22,7-77,3$ & 25 & 83,3 & $65,3-94,4$ & \\
\hline \multicolumn{8}{|l|}{ ESTADO CIVIL } \\
\hline Solteiro(a) & 1 & 3,3 & $0,1-17,2$ & 3 & 10,0 & $2,1-26,5$ & $0,05^{\star}$ \\
\hline Casado(a) & 18 & 60,0 & $40,6-77,3$ & 11 & 36,7 & $19,9-56,1$ & \\
\hline Viúvo(a) & 11 & 36,7 & $19,9-56,1$ & 11 & 36,7 & $19,9-56,1$ & \\
\hline Separado(a) & 0 & 00,0 & - & 5 & 16,7 & $5,6-34,7$ & \\
\hline \multicolumn{8}{|l|}{ RAÇA/COR } \\
\hline Branca & 13 & 43,3 & $25,5-62,6$ & 18 & 60,0 & $40,6-77,3$ & 0,21 \\
\hline Parda & 9 & 30,0 & $14,7-49,4$ & 9 & 30,0 & $14,7-49,4$ & \\
\hline Negra & 8 & 26,7 & $12,3-45,9$ & 3 & 10,0 & $2,1-26,5$ & \\
\hline
\end{tabular}

* Qui-quadrado não válido. ${ }^{* \star}$ Estatisticamente significante em nível de $5 \%$ 
Em relação ao estado civil dos idosos, $60 \%$ dos participantes de grupo são casados(as) e $36,7 \%$ são viúvos(as); já entre os idosos não-participantes foi encontrado percentual semelhante de $36,7 \%$ de casado(a) e viúvo(a).

$\mathrm{Na}$ variável raça/cor prevaleceu a branca $43,3 \%$ de idosos participantes de grupo e $60,0 \%$ de idosos não-participantes.

Quanto à análise das variáveis sociais, não foi encontrada diferença estatisticamente significante entre os grupos; no entanto, na análise das frequências absoluta e relativa, observa-se para escolaridade uma porcentagem grande de idosos analfabetos, tanto entre os participantes de grupo quanto entre os não-participantes: 36,7\% e 40,0\%, respectivamente. Além disso, pode-se observar que os idosos não-participantes de grupo possuem menos anos de estudo (36,7\% possuem de um a três anos); entre os participantes de grupo 36,7\% possuem de quatro a sete anos de estudo. Grande parte dos idosos participantes ou não de grupo vive em companhia de uma ou duas pessoas: $60,0 \%$ e $53,3 \%$, respectivamente; em relação à renda, $80,0 \%$ dos idosos em geral recebe de um a dois salários mínimos e são aposentados; não trabaIham mais fora de casa $90,0 \%$ dos idosos participantes de grupo e 93,3\% idosos não-participantes (Tabela 2).

Tabela 2 - Distribuição percentual de idosos segundo variáveis sociais, Camaragibe, 2010

\begin{tabular}{|c|c|c|c|c|c|c|c|}
\hline \multirow[t]{2}{*}{ VARIÁVEL } & \multicolumn{3}{|c|}{ PARTICIPANTE DE GRUPO } & \multicolumn{3}{|c|}{$\begin{array}{l}\text { NÃO PARTICIPANTE DE } \\
\text { GRUPO }\end{array}$} & \multirow{2}{*}{$\begin{array}{l}\text { VALOF } \\
\text { DE P }\end{array}$} \\
\hline & $\mathbf{n}$ & $\%$ & I.C. $(95 \%)$ & $\mathbf{n}$ & $\%$ & I.C. (95\%) & \\
\hline \multicolumn{8}{|l|}{ ESCOLARIDADE } \\
\hline Analfabeto & 11 & 36,7 & $19,9-56,1$ & 12 & 40,0 & $22,7-59,4$ & $0,01^{*}$ \\
\hline $1-3$ anos & 3 & 10,0 & $2,1-26,5$ & 11 & 36,7 & $19,9-56,1$ & \\
\hline 4-7 anos & 11 & 36,7 & $19,9-56,1$ & 7 & 23,3 & $9,9-42,3$ & \\
\hline+7 & 5 & 16,7 & $5,6-34,7$ & 0 & 00,0 & - & \\
\hline \multicolumn{8}{|l|}{$\begin{array}{l}\text { № DE PESSOAS NO } \\
\text { DOMICÍLIO }\end{array}$} \\
\hline Sozinho & 2 & 6,7 & $0,8-22,1$ & 8 & 26,7 & $12,3-45,9$ & $0,11^{*}$ \\
\hline $2-3$ & 18 & 60,0 & $40,6-77,3$ & 16 & 53,3 & $34,3-71,7$ & \\
\hline $4-5$ & 8 & 26,7 & $12,3-45,9$ & 6 & 20,0 & $7,7-38,6$ & \\
\hline $6+$ & 2 & 6,7 & $0,8-22,1$ & 0 & 00,0 & - & \\
\hline \multicolumn{8}{|l|}{ RENDA } \\
\hline Nenhuma & 1 & 3,3 & $0,1-17,2$ & 1 & 3,3 & $0,1-17,2$ & $0,94^{\star}$ \\
\hline $\begin{array}{l}\text { Menos de } 1 \text { salário } \\
\text { mínimo }\end{array}$ & 3 & 10,0 & $2,1-26,5$ & 2 & 6,7 & $0,8-22,1$ & \\
\hline $\begin{array}{l}1 \text { a } 2 \text { salários } \\
\text { mínimos }\end{array}$ & 24 & 80,0 & $61,4-92,3$ & 24 & 80,0 & $61,4-92,3$ & \\
\hline $\begin{array}{l}\text { Mais de } 2 \text { salários } \\
\text { mínimos }\end{array}$ & 2 & 6,7 & $0,8-22,1$ & 3 & 10,0 & $2,1-26,5$ & \\
\hline \multicolumn{8}{|l|}{ TRABALHO } \\
\hline Não trabalha & 27 & 90,0 & $73,5-97,9$ & 28 & 93,3 & $77,9-99,2$ & 0,50 \\
\hline Trabalha & 3 & 10,0 & $2,1-26,5$ & 2 & 6,7 & $0,8-22,1$ & \\
\hline \multicolumn{8}{|l|}{ APOSENTADORIA } \\
\hline Não & 6 & 20,0 & $7,7-38,6$ & 6 & 20,0 & $7,7-38,6$ & 1,0 \\
\hline Sim & 24 & 80,0 & $61,4-92,3$ & 24 & 80,0 & $61,4-92,3$ & \\
\hline
\end{tabular}

* Qui-quadrado não válido 
$\mathrm{Na}$ análise da capacidade funcional e da linguagem dos idosos avaliados também não foram encontradas diferenças estatisticamente significantes entre idosos participantes e não-participantes de grupo.

Não foram encontrados idosos dependentes totais das atividades instrumentais de vida diária; pois um dos pontos de exclusão foi a incapacidade motora de locomoção, assim era de se esperar que nenhum dos idosos entrevistados tivesse dependência total. Observa-se ainda que o percentual de idosos dependentes parciais prevaleceu entre os idosos não participantes de grupo $(20 \%)$, em comparação aos idosos participantes (10\%). Assim, os idosos que participam de grupos de convivência são mais independentes que os idosos não- participantes de grupos (Tabela 3).

Tabela 3 - Distribuição percentual de idosos segundo dependência de AIVD e de linguagem, Camaragibe, 2010

\begin{tabular}{|c|c|c|c|c|c|c|c|}
\hline \multirow[t]{2}{*}{ VARIÁVEL } & \multicolumn{3}{|c|}{ PARTICIPANTE DE GRUPO } & \multicolumn{3}{|c|}{$\begin{array}{c}\text { NÃO PARTICIPANTE DE } \\
\text { GRUPO }\end{array}$} & \multirow{2}{*}{$\begin{array}{c}\text { VALOR } \\
\text { DE P }\end{array}$} \\
\hline & $\mathbf{n}$ & $\%$ & I.C. (95\%) & $\mathbf{n}$ & $\%$ & I.C. $(95 \%)$ & \\
\hline \multicolumn{8}{|c|}{ DEPENDÊNCIA DE AIVD } \\
\hline Dependência total & 0 & 00,0 & - & 0 & 00,0 & - & 0,23 \\
\hline Dependência parcial & 3 & 10,0 & $2,1-26,5$ & 6 & 20,0 & $7,7-38,6$ & \\
\hline Independência & 27 & 90,0 & $73,5-97,9$ & 24 & 80,0 & $61,4-92,3$ & \\
\hline \multicolumn{8}{|l|}{ LINGUAGEM } \\
\hline Com déficit & 3 & 10,0 & $2,1-26,5$ & 2 & 6,7 & $0,8-22,1$ & 0,50 \\
\hline Sem déficit & 27 & 90,0 & $73,5-97,9$ & 28 & 93,3 & $77,9-99,2$ & \\
\hline
\end{tabular}

No entanto, ao se analisar a linguagem dos participantes, foi encontrado um percentual mais elevado de idosos com déficit de linguagem entre os participantes de grupo (10\%) em comparação aos não participantes $(6,7 \%)$.

\section{DISCUSSÃO}

A pesquisa traz resultados compatíveis com outros estudos em relação às variáveis demográficas. A faixa etária prevalente foi a de 60-69 anos, a qual se assemelha aos dados encontrados em pesquisas relacionadas ao envelhecimento ${ }^{18-20} . \mathrm{Na}$ pesquisa não foram identificados idosos com mais de 90 anos, o que existe na população brasileira, com uma frequência de $1,8 \%$, segundo os dados do $\mathrm{IBGE}^{21}$. Isso mostra que a área geográfica pesquisada possui características de uma ocupação recente; uma vez que as áreas de abrangência das Unidades de Saúde onde foi realizada a coleta dos dados possuem aproximadamente 70 anos de ocupação, prevalecendo na região uma população de adultos, jovens e crianças.

Em relação ao sexo, foi encontrada uma porcentagem superior para o sexo feminino, correspondente à porcentagem do território nacional que tem para a população idosa um percentual de $60,0 \%$ feminino e 40,0\% masculino ${ }^{21}$. No entanto, esse resultado é semelhante apenas para os idosos participantes de grupo, pois o valor achado para o sexo feminino entre os idosos não-participantes é bem superior, ultrapassando $80,0 \%$. Sendo esta a única variável com diferença significante no estudo ( $p$ 0,04). Não foi encontrada nenhuma pesquisa com essa elevada taxa de porcentagem para o sexo feminino em detrimento ao masculino na população idosa, essa predominância de mulheres pode indicar uma maior sobrevida. Esse fato pode ser decorrente de condutas menos agressivas das mesmas, exposição reduzida a riscos no ambiente e, diante de problemas de saúde, elas ficam mais atentas e procuram os serviços de saúde disponíveis ${ }^{22}$.

Na variável estado civil, prevalece a condição de casado(a), seguido de viúvo(a). Em estudo ${ }^{23}$ realizado com idosos de uma área de abrangência de uma Estratégia Saúde da Família, em Ribeirão Preto-SP, encontra-se resultados diferentes, prevalecendo o estado de viúvo(a) com $48,8 \%$ e depois casado(a) com $45,3 \%$. Já em outro estudo ${ }^{24}$, as categorias se invertem, predominando casado(a) com $49,5 \%$ e depois viúvo(a) com $34,7 \%{ }^{19}$, porém esses valores são inferiores ao encontrado no atual estudo que foi de $60,0 \%$ de idosos casado(a). Em uma pesquisa mais recente foi encontrado um 
percentual de casado(a) que chega a 70,0\%. Esse resultado se aproxima mais do presente estudo. Essas porcentagens podem indicar que os idosos conseguem viver por mais tempo em união estável. Esse fato mostra que viver com um parceiro pode trazer mais segurança e benéficios para a saúde dos idosos.

A pesquisa mostrou um percentual maior de idosos da raça/cor branca, esse resultado é semeIhante a estudos ${ }^{24}$ realizados em São Paulo, com taxas em média de $45,0 \%$. Outro estudo ${ }^{25}$ realizado na cidade de São Paulo, referente à qualidade de vida e estado nutricional de idosos, mostrou que $70 \%$ dos pesquisados se declararam de cor branca, demonstrando que o Brasil se formou pela miscigenação de raças e que estas são, predominantemente, de origem europeia. Ainda pode-se citar o fenômeno da migração, que fizeram muitos nordestinos, incluindo pernambucanos, irem para o estado de São Paulo em busca de melhores condições de vida e trabalho, esse fato aproxima os dois estados, podendo ser uma das razões para que os dados entre os dois estados sejam semelhantes.

Quanto às variáveis socioeconômicas dos idosos, foi observada uma elevada taxa de analfabetos e idosos com poucos anos de estudo. A baixa escolaridade encontrada se aproxima de outros estudos $^{26,27}$, que em média apresentaram uma porcentagem $35,2 \%$ de idosos analfabetos. Idosos com maior nível de escolaridade são mais independentes no autocuidado, incluindo o uso correto de medicamentos, de meios de transporte e comunicação ${ }^{28}$. Assim, os idosos analfabetos podem estar mais sujeitos a dificuldades nas atividades de vida diária. Baixos níveis de educação e piores condições sócioeconômicas estão associados a maiores riscos de deficiência e morte ${ }^{29}$. Oportunidades de aprendizado podem ajudar as pessoas a desenvolver as habilidades e confiança para se adaptar e desenvolver um processo de envelhecimento saudável. Idosos com melhores condições financeiras geralmente possuem melhor acesso à prevenção, tratamento e reabilitação.

No arranjo familiar, prevalecem idosos que moram acompanhados. Um estudo ${ }^{26}$, relativamente recente, realizado em Belo Horizonte, verificou que uma porcentagem de $98,98 \%$ de idosos moram pelo menos com mais uma pessoa, o estudo também faz uma distribuição: 34,0\% moram com filho(a) s e $28,43 \%$ moram com o(a) companheiro(a). A família, tanto quanto a comunidade tem um lugar de destaque na criação de uma estrutura que estimula novos caminhos para o idoso ${ }^{30}$, ou seja, além da família, o convívio em sociedade permite a troca de carinho, experiências, idéias, sentimentos, conhecimentos, dúvidas, além de uma troca permanente de afeto.

Nessa pesquisa a taxa de idosos não-participantes de grupo que mora sozinho foi de $26,7 \%$, um valor que deve ser considerado e discutido, pois o fato de morar sozinho constitui uma situação de risco, isso numa visão protecionista do envelhecimento, principalmente se o idoso possui algum comprometimento funcional, tendo-se em vista a possibilidade de perda de autonomia e inadequado suporte familiar ${ }^{31}$. Os idosos que vivem sem companhia necessitam de boa qualidade de vida para que possam desempenhar suas atividades de forma independente, eles precisam de atenção e cuidados que vão além da assistência à saúde, como por exemplo, o convívio familiar e social.

A renda do idoso é um dos principais determinantes do seu estado de saúde. Em geral, idosos com renda mais baixa apresentam piores condições de saúde, de função física e menor uso de serviços de saúde ${ }^{32}$. No presente estudo foram identificados que $80,0 \%$ dos idosos têm renda de um a dois salários mínimos, esse resultado se aproxima de outra pesquisa ${ }^{33}$ realizada no nordeste brasileiro, em que a maior percentagem, cerca de $37,2 \%$ de idosos recebem até dois salários mínimos. Este fato mostra que a população desse estudo é desprovida economicamente de meios que propiciem um bem-estar nessa fase da vida, a qual demanda de tantos gastos com saúde, moradia, alimentação e lazer, afetando sensivelmente sua qualidade de vida.

Também é possível verificar que a renda está intimamente relacionada ao estado de aposentadoria do idoso, que obteve a mesma porcentagem de $80,0 \%$ para idosos participantes e não-participantes de grupo de convivência. É possível ainda associar com a ocupação profissional, em que mais de $90,0 \%$ dos idosos não trabalham mais fora de casa, esse resultado foi discordante do estudo $^{33}$, realizado no Espírito Santo com idosos atendidos em unidades de saúde da família, que revela que $13,0 \%$ dos idosos continuavam tendo uma ocupação profissional. Esta realidade se dá pela necessidade de complementação de renda, visto que com a aposentadoria o idoso tem que se adaptar ao declínio econômico, impondo ao mesmo uma redefinição do padrão de vida. Esse fato leva a sugerir que o idoso funciona, também, como uma fonte geradora de recursos para a família ${ }^{34}$.

O perfil dos idosos quanto às variáveis demográficas e socioeconômicas descritas pode estar diretamente associado ao grau de independência do idoso. Um estudo mostrou que, quanto maior a renda familiar, o grau de escolaridade e melhor estado geral de saúde, menor a necessidade de cuidador ${ }^{35}$. 
A presente pesquisa mostra que a grande parte dos idosos pesquisados se mostrou independente $(90,0 \%$ e $80,0 \%$ para idosos participantes e não-participantes de grupo respectivamente) na maioria das atividades instrumentais de vida diária analisadas. No entanto, não é possível generalizar os dados obtidos a toda uma população de idosos porque a investigação foi realizada com pessoas idosas, porém saudáveis.

Esse resultado não é compatível em relação a outro estudo $^{19}$, que identificou apenas $27,4 \%$ de idosos independentes, 68,4\% com dependência parcial e 4,2\% apresentavam dependência total, perfazendo $72,6 \%$ de idosos com dificuldades para administrar o ambiente em que vivem e lidar com a vida social de forma autônoma. Esse grau de dependência para as atividades instrumentais de vida diária foi encontrado em outro estudo no valor de 58,0\% com dependência em uma ou mais atividades $^{36}$. Outro estudo ${ }^{37}$ em relação às AIVD's obteve uma porcentagem de $58,1 \%$ de idosos que possuíam algum grau de dependência, para administrar o seu ambiente e lidar com a vida social, sendo $57,1 \%$ com dependência parcial e $1 \%$ com dependência total.

As limitações, dependência/independência, adaptações na realização de muitas atividades pelos idosos podem decorrer do processo de envelhecimento ou da evolução de doenças crônico-degenerativas ${ }^{37}$.

A capacidade funcional do idoso consiste em importante indicador do grau de independência, bem como da necessidade de medidas preventivas ou mesmo de intervenções terapêuticas que reduzam os mecanismos que afetam o declínio da habilidade de o indivíduo exercer diversas funções físicas e mentais cotidianas ${ }^{38}$.

Atualmente, as pesquisas voltadas ao envelhecimento têm enfatizado a necessidade da implementação de serviços de promoção à saúde, preservação da vida e recuperação de doenças, de modo que a capacitação das pessoas para cuidar de idosos se reflita no desempenho do autocuidado, na manutenção da autonomia e independência dos idosos ${ }^{2}$.

Nesta pesquisa não foram encontradas diferenças significantes em relação às atividades instrumentais de vida diária de idosos participantes e não-participantes de grupo ( $p 0,23$ ), no entanto, idosos que frequentam grupos de convivência com outras pessoas redescobrem-se, trocam, vivem, sonham e ajudam-se e, isso contribui para a socialização, para manter e adaptar por mais tempo possível a independência física, mental e social, e ainda, reconstruir padrões de vida e atividades ${ }^{39}$. A participação em grupos influencia positivamente na qualidade de vida dos idosos ${ }^{18}$.

Os grupos representam tanto um espaço de educação em saúde como uma fonte de estímulo à organização local, pois facilitam o exercício da cidadania, por meio de projetos comunitários. Constituem-se em alternativa para que as pessoas retomem papéis sociais e/ou outras atividades de ocupação do tempo livre (físicas, de lazer, culturais ou de cuidado com o corpo e a mente) e o relacionamento interpessoal e social. Agregam pessoas com dificuldades semelhantes e possibilitam 0 convívio ${ }^{15}$.

Em relação à linguagem, o resultado foi correspondente ao grau de dependência parcial para as Atividades Instrumentais de Vida Diária, sendo encontrado déficit em apenas $10,0 \%$ dos idosos participantes de grupo, já entre os idosos não-participantes, 20,0\% apresentaram déficit nas AIVD's e 6,7\% déficit de linguagem. Essa diferença encontrada em relação ao déficit de linguagem entre idosos participantes e não-participantes pode indicar que os idosos que participam de grupos de intervenção são aqueles que possuem dificuldades e estão lá por reconhecimento de que têm necessidades de cuidados dos profissionais de saúde.

Os problemas relacionados ao envelhecimento são caracterizados pela perda da funcionalidade, entre elas a funcionalidade motora tem sido a mais descrita, mas a diminuição nos mecanismos de defesa natural do organismo e de adaptação ao ambiente além de perda da reserva funcional estende-se a outras esferas cognitivas, como a linguagem ${ }^{40}$.

Não foi encontrado nenhum estudo que correlacionasse as Atividades Instrumentais de Vida Diária com a linguagem de idosos. No entanto, observou-se neste estudo uma correspondência entre essas variáveis, podendo indicar que existe uma influência entre elas. Ou seja, as atividades instrumentais de vida diária dependem da linguagem; e se o desempenho dessas atividades estiver prejudicado, a linguagem também poderá sofrer alterações e vice-versa.

O estudo também não conseguiu obter diferenças significantes entre os idosos participantes e não-participantes de grupo ( $p 0,50)$, esse resultado pode ser decorrente de uma amostra populacional pequena e/ou de diversos fatores que podem influenciar no resultado, uma vez que os determinantes da incapacidade funcional são multifatoriais. A presença dos mesmos fatores de risco para limitação funcional em indivíduos diferentes pode gerar manifestações diversas, com diferentes repercussões nas atividades diárias. $\mathrm{O}$ ambiente social e físico e os fatores emocionais, econômicos e de 
saúde interagem na expressão de todo o potencial funcional do idoso ${ }^{41}$.

$\mathrm{Na}$ interpretação dos resultados do presente estudo é importante levar em consideração as limitações metodológicas. A amostra de 60 indivíduos foi considerada insuficiente para a análise estatística dos dados. Algumas caselas de tabelas contiveram menos de 5 indivíduos, o que impossibilitou a realização de alguns testes estatísticos. Também é importante considerar as limitações do desenho de estudo aplicado, por se tratar de um estudo transversal, não houve possibilidade de controlar algumas variáveis, o que pode ter influenciado na não significância encontrada na maioria das variáveis analisadas.

\section{CONCLUSÃO}

Por meio da análise da capacidade funcional e da linguagem dos idosos participantes e não-participantes de grupo, puderam-se identificar aspectos relevantes para a qualidade de vida desses indivíduos no que se refere à sua independência e comunicação efetiva, isso contribui para o desenvolvimento de estratégias e ações, na tentativa de interferir no estilo de vida individual, transformando a qualidade de vida do avaliado, como também de toda a população idosa.

Há necessidade de estudos com amostras populacionais maiores na tentativa de comprovar os efeitos positivos das atividades de grupo, como também novos estudos sobre a influência da linguagem na capacidade funcional dos idosos, a fim de encontrar relevância estatística dos dados e assim poder planejar e melhorar o atendimento às necessidades e os problemas decorrentes do envelhecimento.

\section{AGRADECIMENTOS}

Aos idosos participantes desta pesquisa, pela colaboração e pelos momentos compartilhados no grupo.

Às Agentes Comunitárias de Saúde - Grinaura Maria, Josileide Maria, Socorro Josefa, Lucenita Maria, Maria de Fátima e Simone Franca, que contribuíram para a efetivação desse estudo, por meio da ajuda na coleta.

\begin{abstract}
Purpose: to check out and compare the functional ability and the language of participants and nonparticipating elderly in groups of multidisciplinary intervention in primary health. Method: studied participants were 60 subjects over 60 year old of both genders, resident in the coverage of both units of Family Health in the City of Camaragibe/PE. The study was a descriptive, observational, cross one and was conducted from May to September 2010. To collect the data we used a socioeconomic and demographic pre-structured questionnaire, and protocols for assessing the Instrumental Activities of Daily Living scale and Mini-Mental State Examination in order to assess language status. Results: most of the patients are evaluated in the age group of 60-69 year-old female Caucasian, married, an illiterate, and income of two minimum wages. In assessing the functional ability of participant and nonparticipants elderly groups, $90 \%$ and $80 \%$ are independent from the instrumental activities of daily live, respectively. In relation to language, $10 \%$ of elderly participants in the group showed a deficit compared with only $6.7 \%$ of elderly non-participants. Conclusion: we need more studies with larger population samples in an attempt to demonstrate the positive effects of group activity, as per literature, and still further studies on the influence of language on the functional capacity of elderly, to find statistical relevance of data and so we may be able to plan and better meet the needs and problems of aging.
\end{abstract}

KEYWORDS: Aged; Language; Primary Health Care 


\section{REFERÊNCIAS}

1. Vecchia RD, Ruiz T, Bocchi SCM, Corrente JE. Qualidade de vida na terceira idade: um conceito subjetivo. Rev Bras Epidemiol. 2005; 8(3): 246-52.

2. Brasil. Ministério da Saúde. Secretaria de Atenção à Saúde. Departamento de Atenção Básica. Envelhecimento e saúde da pessoa idosa / Ministério da Saúde, Secretaria de Atenção à Saúde, Departamento de Atenção Básica - Brasília: Ministério da Saúde, 2006.

3. Brasil. Ministério da Saúde. Política Nacional de Saúde do Idoso. Portaria № 1.395. Brasília, p.6, 1999.

4. Santos RL, Virtuoso JSJ. Confiabilidade da versão brasileira da Escala de Atividades Instrumentais da Vida Diária. RBPS. 2008; 21(4): 290-6.

5. Brasil. Política Nacional de Saúde da Pessoa Idosa. Portaria n. 2.528. Brasília, 2006.

6. Flangola A, Bezerra BAF, Santo ACGE, Batista Filho M. Concepções e Práticas de agente comunitário na atenção à saúde do idoso. Rev. Saúde Pública. 2005; 39(5):809-15.

7. Brasil. Ministério da saúde. Gabinete do Ministro. Portaria n.702, 12 abr. 2002. Cria mecanismos para organização e implantação de Redes Estaduais de Assistência à Saúde do Idoso. Brasília: Ministério da Saúde, 2002.

8. Brasil. Ministério da saúde. Secretaria de Assistência à Saúde. Portaria n. 249, 16 abr. 2002 Aprova as normas para cadastramento de Centros de Referência em Assistência à Saúde do Idoso. Brasília: Ministério da Saúde, 2002.

9. Brasil. Ministério da saúde. Secretaria de Atenção à Saúde - Departamento de Atenção Básica. Portaria № 154/GM, de 24 de janeiro de 2008.

10. Molini-Avejonas DR, Mendes VLF, Amato CAH. Fonoaudiologia e Núcleos de Apoio à Saúde da Família: conceitos e referências. Rev Soc Bras Fonoaudiol. 2010;15(3):465-74.

11. Oliveira DL. A 'nova' saúde pública e a promoção da saúde via educação: entre a tradição e a inovação. Revista Latino Americana Enfermagem. 2005; 13(3):423-31.

12. Zimerman GI. Grupos com idosos. In: Zimerman DE, Osório LC. Como trabalhamos com grupos. Porto Alegre: Editora Artes Médicas; 1997.

13. Souza APR, Crestani AH, Vieira CR, Machado FCM, Pereira LL. O Grupo na Fonoaudiologia: Origens Clínicas e na Saúde Coletiva. Rev. CEFAC, São Paulo. 2011; jan.-fev. 13(1):140-51.

14. Mendes VLF. Grupos e Processos Grupais. In: Tomé MC(org). Dialogando com o coletivo Dimensões da saúde em Fonoaudiologia. São Paulo: Editora Santos; 2009. p. 107-18.
15. Garcia MAA, Yagi GH, Souza CS, Odoni APC, Frigério RM, Merlin SS. Atenção à saúde em grupos sob a perspectiva dos idosos. Rev. LatinoAmericana de Enfermagem. 2006; 14(2):175-82.

16. Freitas EV, Miranda RD. Parâmetros clínicos do envelhecimento e Avaliação geriátrica ampla. In: Freitas EV. Tratado de Geriatria e Gerontologia. $2^{a}$ ed. Rio de Janeiro: Guanabara-Koogan; 2006. p. 900-9.

17. Folstein MF, Folstein SE, McHugh PR. Minimental state: a practical method for grading the cognitive state of patients for the clinician. J Psychiatric Res. 1975;12:189-98.

18. Coutinho RX, Santos CM, Sanguin CMR, Acosta MAF. Perfil de idosos praticantes de atividades físicas: saúde e atividades da vida diária. Revista Digital - Buenos Aires [internet]. Jun 2009 [acesso em nov 2010]; 14(133):[6]. Disponível em: http:// www.efdeportes.com/.

19. Costa EC, Nakatani AYK, Bachion MM. Capacidade de idosos da comunidade para desenvolver Atividades de Vida Diária e Atividades Instrumentais de Vida Diária. Acta Paul Enferm. 2006;19(1):43-8.

20. Ursine PGS, Moraes CL. Prevalência de Idosos Restritos ao Domicílio em região metropolitana de Belo Horizonte, Minas Gerais. Rev Ciência \& Saúde Coletiva. 2011;16(6):2953-62.

21. IBGE: Instituto Brasileiro de Geografia e Estatística. Censo Demográfico 2000. Perfil dos idosos responsáveis pelos domicílios no Brasil 2000. Tabela1 - População residente, total e de 60 anos ou mais de idade, por sexo e grupos de idade, segundo as Grandes Regiões e Unidades da Federação - 1991/2000. Brasília (DF); 2000. Disponível em: URL:<http://www.ibge.com.br/ home/estatistica/populacao/perfilidoso/tabela1_6. shtm>. Acessado em: 03 nov 2010.

22. Araújo MAS, Nakatani AYK, Silva LB, Bachion MM. Perfil do idoso atendido por um programa de saúde da família em Aparecida de Goiânia - GO. Revista da UFG [internet]. Dez 2003 [acesso em nov 2010]; 5(2): [5]. Disponível em: URL: <http:// www.proec.ufg.br/revista_ufg/idoso/perfil.html>

23. Farinasso ALC. Perfil dos idosos em uma área de abrangência da estratégia de saúde da família [dissertação]. Ribeirão Preto (SP): Escola de Enfermagem de Ribeirão Preto da Universidade de São Paulo; 2005.

24. Stumm EMF, Zambonato D, Kirchner RM, Dallepiane LB, Berlezi EM. Perfil de idosos assistidos por unidades de Estratégia de Saúde da Família que sofreram infarto agudo do miocárdio. Rev. Bras. Geriatr. Gerontol. 2009; 12(3):449-61. 25. Quintella LCM. Qualidade de vida e estado nutricional de idosos: um estudo 
descritivo sobre freqüentadores do Centro de Práticas Esportivas da Universidade de São Paulo [dissertação]. São Paulo (SP): Universidade de São Paulo; 2006.

26. Borges PLC, Bretas RP, Azevedo SF, Barbosa JMM. Perfil dos idosos freqüentadores de grupos de convivência em Belo Horizonte, Minas Gerais, Brasil. Cad. Saúde Pública. 2008; 24(12):2798-808. 27. Leal MCC, Marques APO, Marino JG, Rocha EC, Santos CR, Austregésilo SC. Perfil de pacientes idosos e tempo de permanência em ambulatório geronto-geriátrico. Rev. Bras. Geriatr. Gerontol. 2009; 12(1):77-86.

28. Lemos M, Souza NR, Mendes MMR. Perfil da população idosa cadastrada em uma unidade de saúde da família. REME- Revista Mineira de Enfermagem. 2006; 10(3): 218-25.

29. Organização Mundial da Saúde. Envelhecimento ativo: uma política de saúde. Brasília: Organização Pan-Americana da Saúde; 2005.

30. Mendes MRSSB, Gusmão JL, Faro ACM, Leite RCBO. A situação social do idoso no Brasil: uma breve consideração. Acta Paul Enferm. 2005;18(4):422-6

31. Meireles VC, Matsuda LM, Coimbra JAH, Mathias TAF. Características dos idosos em área de abrangência do Programa Saúde da Família na Região Noroeste do Paraná: Contribuições para a gestão do cuidado em enfermagem. Saúde e Sociedade. Saúde e Sociedade. 2007; 16(1):69-80. 32. Lima-Costa MF, Barreto S, Giatti L, Uchoa E. Desigualdade social e saúde entre idosos brasileiros: um estudo baseado na Pesquisa Nacional por Amostra de Domicílios. Cad Saúde Pública. 2003; 19:745-57.

33. Cesar JA, Oliveira JA, Bess G, Cegielka R, Machado J, Gonçalves TS et al. Perfil dos idosos residentes em dois municípios pobres das regiões Norte e Nordeste do Brasil: resultados de estudo transversal de base populacional. Cad. Saúde Pública. 2008; 24(8):1835-45.
34. Silveira SR, Cabral GTR, Correa LB, Reis LB. Análise do perfil dos idosos atendidos por um programa de saúde da família do bairro Araçás em Vila Velha-ES. Ensaios e Ciência: C. Biológicas, Agrárias e da Saúde. 2008; 12(2):35-47.

35. Giacomim KC, Uchoa E, Firmo JO, LimaCosta MF. Projeto Bambuí: um estudo de base populacional da prevalência e dos fatores associados à necessidade de cuidador entre idosos. Cad Saúde Pública. 2005; 21:80-91.

36. Nakatani AYK, Costa EFA, Teles SA, Silva LB, Rêgo MP, Silva e Souza AC, et al. Perfil sóciodemográfico e avaliação funcional de idosos atendidos por uma

equipe de saúde da família na periferia de Goiânia, Goiás. Rev Soc Bras Clin Méd. 2003; 1(5):131-6.

37. Nakatani AYK, Silva LB, Bachion MM, Nunes DP. Capacidade funcional em idosos na comunidade e propostas de intervenções pela equipe de saúde. Rev. Eletr. Enf. 2009;11(1):144-50.

38. Duca, GFD, Silva MC, Hallal PC. Incapacidade funcional para atividades básicas e instrumentais da vida diária em idosos. Rev. Saúde Pública. 2009; 43(5): 796-805.

39. Leite MT, Cappellari VT, Sonego J. Mudou, mudou tudo na minha vida: experiências de idosos em grupos de convivência no município de ljuí/ RS. Revista Eletrônica de Enfermagem. 2002; 4(1):18-25.

40. Garcia FHA, Mansur LL. Habilidades funcionais de comunicação: idoso saudável. Acta Fisiatr. 2006; 13(2): 87-9.

41. Silva AE, Menezes EAG, Coelho TOA, Moraes EN. Aspectos Bio-Psico-Sociais dos Idosos Institucionalizados na Casa do Ancião da Cidade Ozanan, no Ano de 2005, em Belo Horizonte. 8응 Encontro de Extensão da UFMG; 03 a 08 de out de 2005; Universidade Federal de Minas Gerais. Belo Horizonte; 2005. Disponível em: http://www.ufmg. br/proex/arquivos/8Encontro/Saude_7.pdf

http://dx.doi.org/10.1590/S1516-18462012005000019

RECEBIDO EM: 26/04/2011

ACEITO EM: 11/10/2011

Endereço para correspondência:

Aluísia Guerra Albuquerque

Avenida Governador Paulo Guerra, 98 - Centro

Machados - PE

CEP: $55740-000$

E-mail: guerraalu@yahoo.com.br 